\title{
Visuospatial updating of reaching targets in near and far space
}

\author{
W. Pieter Medendorp ${ }^{1,2, C A}$ and J. Douglas Crawford ${ }^{1,2}$ \\ 'Canadian Institutes of Health Research Group for Action and Perception, Centre for Vision Research, and ${ }^{2}$ Department of Psychology, \\ York University, 4700 Keele Street, Toronto, Ont. M3J IP3, Canada \\ ${ }^{\mathrm{CA}}$ Corresponding Author
}

Received 6 December 200I; accepted 4 February 2002

\begin{abstract}
The brain constructs multiple representations of near and far space but it is unclear which spatial mechanism guides reaching across eye movements in near space. Retinocentric reaching representations are known to exist in parietal cortex, but must be updated during eye movements, in order to remain accurate. In contrast, non-retinal (e.g. muscle-centered) reaching plans in motor cortex do not require updating, and so may provide a more stable encoding mechanism. To test between these, we employed a behavioral test. Subjects briefly foveated a target (located at
\end{abstract}

various depths in near and far space) looked peripherally, then reached toward its remembered location. Surprisingly, subjects did not use the stable non-retinal reaching plan (compared to controls without eye movements). Instead, the intervening eye movements induced a systematic pattern of reaching errors for targets at all depths consistent with updating in a retinal frame. We conclude that a common eye-centered updating mechanism prevails in programming arm movements in both near and far space. NeuroReport 13:633-636 (c) 2002 Lippincott Williams \& Wilkins.

Key words: Arm movements; Far vs near space; Reference frames; Spatial perception; Visuomotor control

\section{INTRODUCTION}

The brain constructs multiple representations of space in order to guide behavior [1-9]. In particular, evidence suggests that far (beyond reach) and near (reachable) space are coded in distinct brain areas using different spatial mechanisms. Areas such as ventral and anterior intraparietal areas [1-3] as well as the parietal reach region [4] encode objects within reaching distance, whereas the frontal eye fields [5] or the lateral intraparietal area $[1,6]$ represent far space (primarily for eye movements). Also, clinical studies of patients with distance-specific spatial neglect, support this division of near and far space [7-9].

These spatial mechanisms can be dissociated with regard to the reference frames they use, and how they are updated across eye movement. Far space appears to be coded in an eye-centered frame, which must be updated across eye movements to remain accurate $[1,5,6]$. However, near space is coded in different coordinate frames including eyecentered [2,4], head-centered [2,3] and other non-retinal [10] frames, depending on the perceptual and motor requirements of the task. Interestingly, even the intention to program an arm movement in near space is already accompanied by activity in precentral and central motor cortices, suggesting a muscle-centered coding scheme $[11,12]$. Such non-retinal programs $[2,3,10-12]$ have no need for updating during eye movements, and so would seem to be the more intuitive choice for guiding reaching movements in near space.

Which spatial mechanism dominates human reaching behavior, across eye movements, in near space? Neurophysiological data suggest that several possible mechanisms coexist, but cannot tell which coding scheme prevails in guiding behavior. Therefore, we employed a recently developed test to address this question [13].

The efficacy of this test is based on the localization errors observed when eye movements intervene between viewing a target and pointing toward its remembered location (Fig. 1, top row). The basic observations for this test are the following: human subjects point accurately to the remembered location of foveal targets (Fig. 1a, control condition) but overestimate the eccentricity of retinally peripheral targets (Fig. 1b, static condition) [14-16].

The crucial test then is based on the errors which occur when subjects point, after an intervening eye movement, toward the location of an initially foveated target (Fig. 1c). Accurate pointing, as in the control condition, would confirm the use of a non-retinal reaching program. However, if intervening eye movements lead to errors like those observed in the static paradigm, this would provide evidence for an updated eye-centered reaching plan [13]. Contrary to our expectations, our test results suggest that eye-centered updating dominates human reaching behavior for both near and far space. 
(a)

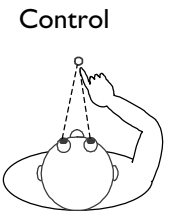

(d)

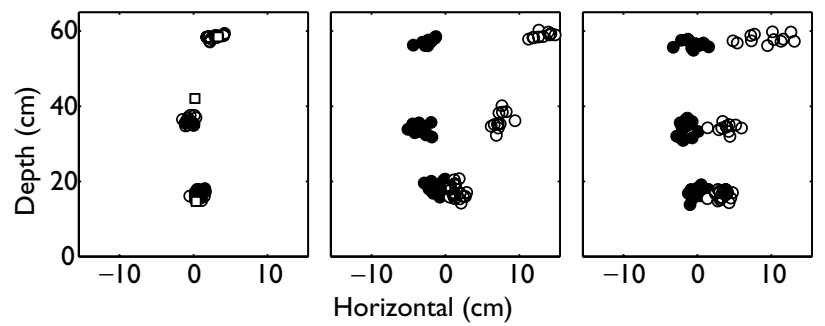

Fig. I. a-c. Illustration of the three paradigms. In the control paradigm (a), subjects viewed the target and either pointed or reached toward the remembered location after it was extinguished. In the static paradigm (b), subjects either pointed or reached to the remembered location of a retinal peripheral (but craniotopically central) target. In the dynamic para$\operatorname{digm}(\mathrm{c})$, subjects either pointed or reached, after an intervening eye movement, toward the remembered location of an initially foveally viewed target. d-f. Final fingertip positions (circles) in the horizontal plane of one subject in the control (d), static (e) and dynamic (f) paradigm. Squares in $D$ represent the actual target locations of the two reaching targets and the fingertip location for pointing toward the continuously illuminated pointing target. In $\mathrm{E}$ and $\mathrm{F}$ open circles indicate 20 leftward eye fixation; solid circles represent data for 20 rightward eye fixation. Eye movements are not shown.

\section{MATERIALS AND METHODS}

All experiments were approved by the York University Human Participants Review Subcommittee. Six subjects (age 22-34 years), with no history of sensory, perceptual or motor disorders, gave informed consent to participate in the experiments. Subjects were seated in complete darkness with the head mechanically stabilized by a personalized bite-bar with their right arm (used for either pointing or reaching) resting unencumbered on their lap. Eye movements were measured using the search coil technique [13]. An OPTOTRAK 3020 system measured the 3-D locations of the eyeballs, shoulder and index fingertip, as well as the positions of the pointing/reaching targets in space. Data were sampled at $100 \mathrm{~Hz}$ and saved on a PC for offline analysis.

Three visual targets (T) (LEDs) were positioned at different distances, $2 \mathrm{~m}, 42 \mathrm{~cm}$ and $15 \mathrm{~cm}$ in front of the subject's eyes in the transverse plane. The target located $2 \mathrm{~m}$ from the subject provided the pointing target (far space) while the remaining two targets served as reaching targets (near space). The two near space targets were mounted on a moveable rod so that, after target presentation, the target could be moved (PC controlled) in order to avoid giving any tactile feedback to the subject. For all three targets, we included distractor targets on either side, at a horizontal eccentricity of about $7^{\circ}$. These distractor targets were randomly presented in $11-22 \%$ of the trials, and confirmed that subjects correctly adjusted their arm movements to the remembered targets and did not merely make habitually guided movements. Finally, we positioned four targets at $-20^{\circ},-10^{\circ}, 10^{\circ}$ and $20^{\circ}$ horizontal eccentricity from the pointing target to serve as our standard eye fixation lights (F).

Experimental paradigms: We tested our subjects using the paradigms described briefly in the Introduction and extensively elsewhere [13], the only difference being the target durations and distances. In the first control task, subjects visually fixated a target ( $\mathrm{T}$, illuminated for $1.3 \mathrm{~s}$ ), and either pointed or reached toward the remembered location of $\mathrm{T}$ after it had been extinguished (Fig. 1a). In the second control task (Fig. 1b), the static paradigm, subjects fixated an eccentric light (F). After $0.7 \mathrm{~s}$, T appeared for $1.3 \mathrm{~s}$ and then both lights were extinguished. Subjects then either reached or pointed to $T$, while keeping their gaze fixated at F. In the final dynamic paradigm (Fig. 1c), subjects initially fixated $\mathrm{T}$ (illuminated for $1.3 \mathrm{~s}$ ). After $\mathrm{T}$ was extinguished, $\mathrm{F}$ was illuminated for $0.7 \mathrm{~s}$ and subjects made a saccade toward, and fixated F. Subsequently, after F was extinguished, subjects pointed or reached toward $\mathrm{T}$ while keeping gaze aligned with $\mathrm{F}$.

Target combinations ( $\mathrm{F}$ and $\mathrm{T}$ ) were selected randomly. For each F, 12 trials were conducted using the far and intermediate target distances and 21 trials were run for the near target distance (in both the static and dynamic condition). In the control task, 16 and 28 trials were executed for the far/intermediate target distances and the near target distance, respectively.

For data analysis, pointing responses were converted into a degree scale by taking the polar angle of fingertip position relative to the center of the two eyes in the horizontal plane.

\section{RESULTS}

The results from one subject are shown in the bottom row of Fig. 1, showing the final fingertip locations, in all three conditions, in the horizontal plane. In the control task (Fig. $1 \mathrm{~d}$, arm movements toward a foveally fixated target) the subject performed fairly accurately although some small depth errors were observed for the two reaching targets (Fig. 1d).

Figure 1e illustrates the results of the static test condition (Fig. 1b, arm movements for a peripherally fixated target) by showing fingertip positions to the three targets when the eye is fixating $20^{\circ}$ right (filled circles) or $20^{\circ}$ left (open circles). Clearly, for both reaching and pointing movements, fingertip positions are displaced compared to the control response (Fig. 1d), depending on the fixation direction. Note also that the fingertip positions strongly suggest an angular pattern of errors as they disperse for farther distances.

For the pointing target, the mean directional error (across subjects) relative to the control response was $-4.3 \pm 3.6^{\circ}$ (mean \pm s.d.) for $20^{\circ}$ leftward fixation and $6.1 \pm 2.7^{\circ}$ for $20^{\circ}$ rightward fixation. These distinct error distributions were significantly different in a $t$-test $(p<0.001)$. Similarly, for the reaching targets we found distinct error distributions: $-4.7 \pm 3.4^{\circ}$ for $20^{\circ}$ leftward fixation and $4.7 \pm 3.2^{\circ}$ for $20^{\circ}$ rightward fixation. These results were significantly different $(p<0.001)$. In addition, all error distributions (for pointing and reaching) were significantly different from their corresponding control responses $(p<0.05)$. Within the static condition however, reaching and pointing errors did not 
differ significantly $(p>0.38)$ confirming that subjects exaggerated the retinal displacement between current fixation and remembered target direction for both pointing [13] and reaching [14-16].

Finally, we tested this subject in the dynamic test condition (Fig. 1c), where the illuminated target was fixated (as in the control condition) but then gaze was deviated (as in the static paradigm) before the arm movement was executed. If the system were using a non-retinal coding scheme, the intervening eye movement would have no effect, and error distributions, similar to those as found in the control condition, are expected to be observed. However, if the system were using oculocentric updating, this would predict a pattern of errors similar to those in the static condition [13]. As shown in Fig. 1f, oculocentric updating prevails at all three target depths. Data from the dynamic paradigm reveals a pattern of error similar to the static paradigm for both pointing (far space) and reaching (near space).

For the pointing target, the mean directional overshoot (across subjects) was $-3.7 \pm 2.2^{\circ}$ for $20^{\circ}$ leftward fixation and $3.9 \pm 2.1^{\circ}$ for $20^{\circ}$ rightward fixation. For the reaching targets, we found average values of $-6.8 \pm 3.0^{\circ}$ and $2.1 \pm 1.4^{\circ}$ respectively. All values were significantly differ- ent from the corresponding control values $(p<0.05)$. Again, as in the static condition, error distributions were significantly different $(p<0.001)$ for leftward and rightward fixation for both reaching and pointing.

The upper panels of Fig. 2 show the averages and s.d. of horizontal directional errors (across subjects) plotted as a function of horizontal eye displacement for the three target distances. The static paradigm errors (squares) followed the same non-linear function of target displacement reported previously for static pointing toward eccentric targets [1416]. More importantly, the dynamic paradigm errors (circles) followed almost exactly the same pattern for both pointing and reaching.

We then performed a linear regression to quantify the relationship between the static errors and the dynamic errors. A gain of 1 would favor oculocentric updating (dynamic error = static error), whereas a gain of 0 would reflect a non-retinal coding scheme (dynamic error $=$ control error). The bottom panels of Fig. 2 show the regression lines for all six subjects (gray lines) superimposed on the mean regression line (thick line) across subjects for each target. The results clearly favor the oculocentric hypothesis for all target depths. The average slopes were $0.79 \pm 0.20,0.81 \pm 0.30$ and $1.18 \pm 0.37$ for the far,
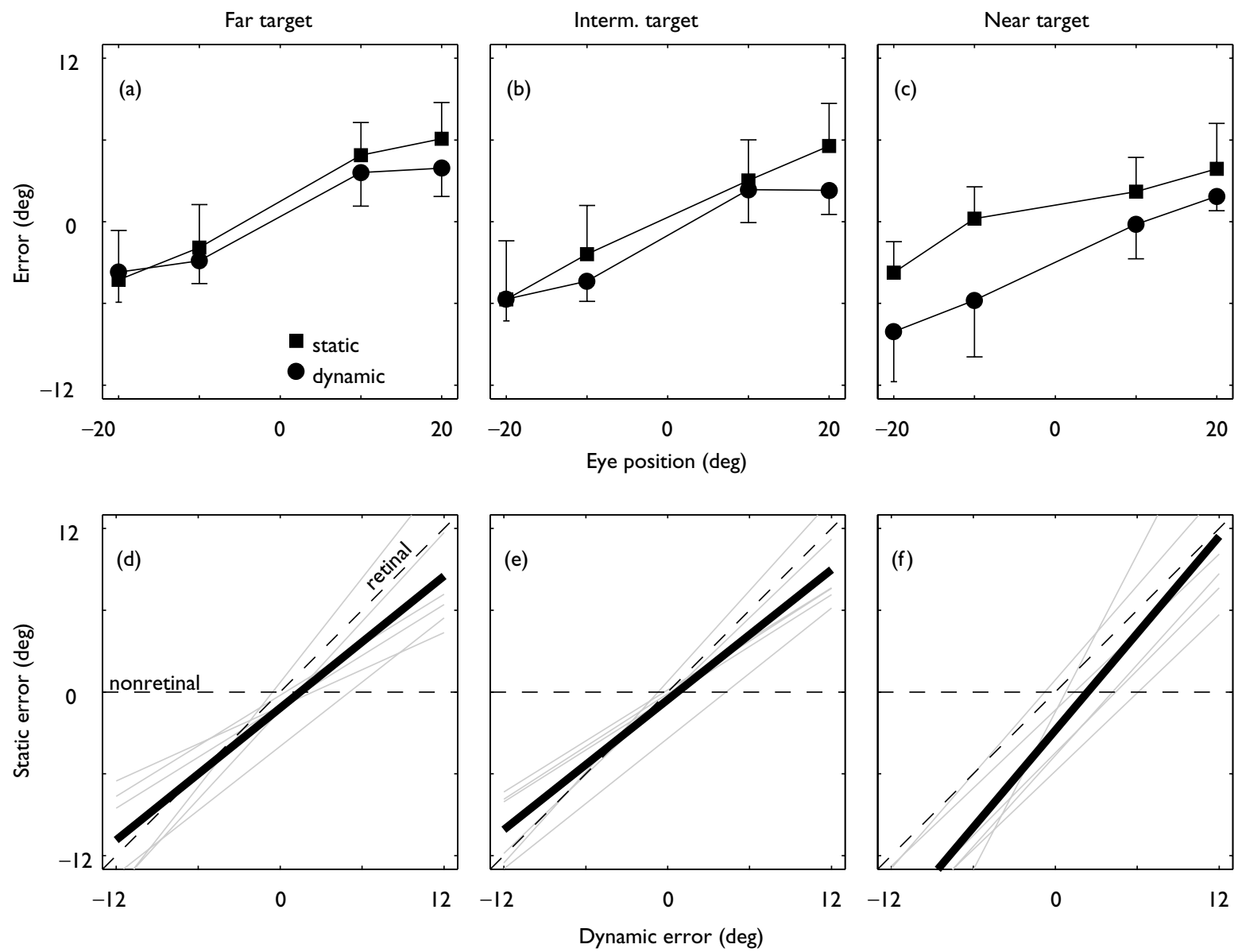

Fig. 2. a-c. Mean directional errors (ñSD) (across subjects) in the static (squares) and dynamic (circles) paradigm plotted as function of angular eye displacement for the three targets. $\mathbf{d}-\mathbf{f}$. Regression lines for all subjects (thin lines) quantifying the relationship between the static and dynamic paradigm errors for the three targets. The average regression line across subjects is given by the thick line. 
intermediate and near targets, respectively. These slopes were significantly different from 0 ( $t$-test, $p<0.002$ ) but were not significantly different from 1 ( $t$-test; $p>0.06$ ), indicating that our results can be best explained by an oculocentric updating principle.

\section{DISCUSSION}

In agreement with previous studies [13-16], our results showed that humans make localization errors when reaching towards remembered locations of retinally peripheral targets in near space. Furthermore, the same pattern of errors was found when the eyes moved after a foveal target appeared but before reaching to the remembered location (compared to controls without eye movements). In other words, subjects did not use a stable non-retinal spatial mechanism to guide the arm movement, for which an intervening eye movement would have had no effect. Instead, our results imply that a common eye-centered spatial mechanism, which is updated across eye movements, guides the arm movement. While this conclusion was previously drawn for pointing in far space [13] we now can say that it also holds for reaching in near space.

We do not argue that there are no head-centered, musclecentered or other non-retinal representations of reach intentions in near space [2,3,10-12]. Our results, however, do suggest that an eye-centered representation dominates during behavior: it updates the non-retinal (e.g. muscle) map even when it is not as accurate. Furthermore, we cannot make the distinction whether this eye-centered updating principle is more relevant for spatial perception $[5,6,17,18]$ than for spatial coding of action [4,19-21]. Since our experiment revealed no differences between reaching in near space and pointing in far space, the near-far distinction may be more relevant for perception than action. If this is true, our results provide strong behavioral support for the idea that target locations are remembered by shifting retinotopic representations as a function of each eye movement, and suggest that this is a general spatial mechanism for near and far space $[6,13,22]$.

\section{CONCLUSION}

While the brain holds different representations for near and far space, we conclude that eye-centered updating mechanisms dominate in human arm movements to targets in both near and far space.

\section{REFERENCES}

1. Colby CL and Goldberg ME. Annu Rev Neurosci 22, 319-349 (1999).

2. Duhamel JR, Bremmer F, BenHamed S et al. Nature 389, 845-848 (1997).

3. Galletti C, Battaglini PP and Fattori P. Exp Brain Res 96, 221-229 (1993).

4. Batista AP, Buneo CA, Snyder LH et al. Science 285, 257-260 (1999).

5. Bruce CJ and Goldberg ME. J Neurophysiol 53, 603-635 (1985).

6. Duhamel JR, Colby CL and Goldberg ME. Science 255, 90-92 (1992).

7. Halligan PW and Marshall JC. Nature 350, 498-500 (1991).

8. Vuilleumier P, Valenza N, Mayer E et al. Ann Neurol 43, 406-410 (1998).

9. Berti A, Smania N and Allport A. Neuroimage 14, 98-102 (2001).

10. Fogassi L, Gallese V, Fadiga L et al. J Neurophysiol 76, 141-157 (1996).

11. Crammond DJ and Kalaska JF. J Neurophysiol 84, 986-1005 (2000).

12. Riehle A and Requin J. J Neurophysiol 61, 534-549 (1989).

13. Henriques DYP, Klier EM, Smith MA et al. J Neurosci 18, 1583-1594 (1998).

14. Bock O. Exp Brain Res 64, 476-482 (1986).

15. Enright JT. Vision Res 35, 1611-1618 (1995).

16. Lewald J and Ehrenstein WH. Vision Res 40, 539-547 (2000).

17. Tian J, Schlag J and Schlag-Rey M. Exp Brain Res 130, $433-440$ (2000).

18. Gottlieb J and Goldberg ME. Nature Neurosci 2, 906-912 (1999).

19. Mushiake H, Tanatsugu Y and Tanji J. J Neurophysiol 78, 567-571 (1997).

20. Snyder LH, Batista AP and Andersen RA. Nature 386, 167-170 (1997).

21. Zhang $M$ and Barash S. Nature 408, 971-975 (2000).

22. Medendorp WP, Smith MA, Tweed DB et al. J Neurosci 22, RC196, 1-4 (2002). 